\author{
INVESTIGACIONES \\ de HISTORIA ECONÓMICA \\ 2006, invierno, número 4. Pp. 51 a 76
}

\title{
Empresas familiares y acuerdos cooperativos en el metal español: el caso de las industrias del alambre de hierro y acero (1880-1974)
}

\section{Family firms and cooperative agreements in the Spanish metal industries: the example of the iron and steel wire industries (1880-1974)}

\author{
PALOMA FERNÁNDEZ PÉREZ \\ Universidad de Barcelona
}

\author{
RESUMEN \\ ABSTRACT \\ En comparación con otros países occidentales, \\ Family ownership, reduced size of \\ la estructura familiar de la propiedad, el \\ tamaño reducido de los establecimientos \\ fabriles y una importante concentración han \\ caracterizado el desarrollo de las empresas \\ españolas del alambre de hierro y acero. En \\ este artículo se muestra, además, cómo los \\ acuerdos a escala regional entre las más \\ importantes empresas familiares del sector \\ facilitaron la cooperación, la escasa necesidad \\ de fusiones y la posibilidad de que algunas \\ familias mantuviesen el control sobre sus \\ empresas durante más de un siglo. \\ PALABRAS CLAVE: Empresas familiares, \\ Cárteles, Industrias del hierro y el acero, España \\ productive centers, and concentration are \\ historical trends that characterize the \\ development of the Spanish iron and steel \\ wire firms in international comparison. \\ Agreements at regional level between the \\ most significant family firms of the sector \\ allowed coordination, which proved to be \\ useful to avoid absorptions and to keep \\ family control in some of the most important \\ firms during more than a century. \\ KEY WORDS: Family firms, Cartels, Iron and \\ Steel Industries, Spain \\ JEL Codes: L14, N83, N84, 014
}




\section{Introducción ${ }^{1}$}

L

a fabricación de alambre y sus manufacturas, cuyos orígenes en España, entre el siglo XVII y mediados del XIX, fueron muy modestos, registró un crecimiento espectacular durante el XX. Se trata de una actividad muy especializada que ha atendido a la demanda de sectores fundamentales de la economía del país: en el siglo XIX, la agricultura (cerramientos, enrejados) y la minería (cedazos y filtros); en el XX, la construcción (barras corrugadas y mallazo), los transportes (muelles de asientos, refuerzos de neumáticos y filtros) y las telecomunicaciones (cables).

Durante el último siglo y medio, el mercado español del alambre de hierro y acero ha estado dominado por algunas empresas familiares de tamaño medio localizadas en regiones septentrionales del país. En los últimos veinte años, la práctica totalidad de las empresas familiares centenarias que habían venido dominando la trefilería en España ha sido absorbida por un gran Holding, Global Steel Wire (GSW), otra empresa familiar. Integrado en el Grupo CELSA de la siderurgia y controlado por la familia catalana Rubiralta Vilaseca, GSW se ha convertido en el primer productor nacional de alambrón y trefilería, así como en el principal abastecedor de barras corrugadas para la construcción en los mercados español, británico y polaco ${ }^{2}$.

El presenta trabajo estudia el período de crecimiento más intenso de las empresas del sector, entre 1880 y 1974, y pretende explicar para este lapso, en el que se difundió en España la II Revolución Tecnológica y avanzó la gran empresa, por qué se mantuvo en la trefilería el capitalismo familiar de tamaño medio y qué mecanismos institucionales facilitaron ese fenómeno.

Las evidencias aportadas permiten destacar, entre los numerosos factores a tener en cuenta, la formación de redes informales y acuerdos institucionales de cooperación entre empresas y empresarios. Por este motivo, ha sido particularmente útil recurrir, en el análisis interpretativo, a algunas teorías y conceptos relativos a grupos

1 Esta investigación, en distintas etapas, ha recibido financiación de los proyectos públicos PB98-1167 y BEC2002-03890, y de un proyecto de la Fundación BBVA (Convocatoria 2003 de Ayudas a la Investigación en Economía y Empresa). Una primera versión del trabajo fue presentada en el VIII Congreso de la Asociación Española de Historia Económica (Santiago de Compostela, 13-16 septiembre de 2005), en la sesión que sobre la formación del tejido empresarial en España en los siglos XIX y XX organizaron Nuria Puig, Eugenio Torres y Matthias Kipping. Agradezco asimismo a los evaluadores anónimos de Investigaciones de Historia Económica sus comentarios.

2 Según los últimos datos disponibles, relativos al primer trimestre de 2005, GSW obtuvo un beneficio neto de 3 millones de euros y un volumen de negocio de 142,4 millones, una rentabilidad que, sin duda, explica la reciente OPA de exclusión de la Bolsa que la familia Rubiralta, como principal propietaria, impulsó en marzo de 2005. Fernández Pérez (en prensa). 
empresariales (Granovetter), redes y cultura empresarial (Casson), y a la historiografía existente sobre empresas familiares en los países occidentales (Rose, Colli). El concepto de red como un entramado articulado de personas, grupos e instituciones, en el que el juego de la confianza mutua permite reducir los costes de información y transacción, ha sido desarrollado por sociólogos y teóricos de la empresa, y aplicado al estudio de sectores dominados por el "capitalismo familiar" en Europa, Asia y Estados Unidos. En nuestro país, hace unos años que estas ideas se han venido difundiendo, sobre todo entre los historiadores de empresas interesados en entidades distintas de la gran corporación gerencial, y atentos a los factores no estrictamente económicos del entorno empresarial que condicionan sus estrategias de crecimiento. Estos estudios sugieren que el concepto de redes aplicado a la historia empresarial complementa al de distrito industrial, aportándole una mayor flexibilidad en la definición y en el alcance de las relaciones interempresariales, las cuales a menudo fluyen fuera del distrito - más delimitado territorialmente- $-\mathrm{y}$ garantizan su existencia ${ }^{3}$. Además, estas ideas procedentes de la sociología, la economía neoinstitucional y la historia empresarial, resaltan la importancia de factores extraeconómicos en el desarrollo de la actividad económica y empresarial. Según ellas, la afinidad política, religiosa, étnica, ideológica, familiar y también de género, actúan como elementos culturales capaces de articular de manera estable entramados empresariales informales o institucionalizados, particularmente eficientes en entornos políticos dominados por grupos locales de poder económico, así como en épocas y territorios en los que el personalismo domina las reglas de la vida pública, como ha sido el caso de España hasta hace unas décadas.

Este trabajo supone una contribución a esta línea de investigación, a través del estudio de los empresarios y empresas más significativos de un sector que, relativamente intensivo en capital, siempre estuvo dominado por la empresa familiar, y que creció en un entorno regional cuya vida pública fue controlada por el personalismo y por grupos de poder locales y regionales. Se identifican algunas de las redes informales y acuerdos institucionales de cooperación más visibles, forjados por los

3 Granovetter y Swedberg (1992); Casson (1999); y Colli, Fernández Pérez y Rose (2003). Los estudios en los campos de la organización de empresas y la sociología son abundantes. Algunos de los más relevantes para el presente trabajo destacan la imprecisión y el eclecticismo en la formulación de conceptos relacionados con la formación de redes, y la importancia estratégica que la cooperación interempresarial tiene para empresas de pequeño y medio tamaño en entornos personalistas basados fuertemente en la confianza. Destacan Eccles y Noria (1992); Oliver (1998); Wasserman y Faust (1994); Cohendet (1998); Ben-Porath (1980); Borth y Arthur (1995), y Robertson y otros (1996). La abundancia de estudios que aplican las ideas de cooperación y de redes interempresariales a la historia económica, la historia social y la historia de la empresa en distintos continentes se ha puesto recientemente de manifiesto en el X Simposio de Historia Económica, Análisis de Redes en Historia Económica (Bellaterra, 27-29 enero de 2005). Particularmente relevantes para la historia empresarial son las reflexiones de Rose (1999); Granovetter y Swedberg (1992), y Casson (1999). 
responsables de las más importantes empresas familiares de la industria del alambre (de hierro y acero) en España entre 1880 y 1974, años caracterizados por la precariedad en la articulación y defensa de los derechos de propiedad y consumo, en los que fue determinante el poder de las élites locales y regionales en la definición de las reglas de la vida económica en España ${ }^{4}$.

No se pretenden explicar aquí todos los elementos que hicieron que la cooperación fuese una alternativa eficiente y complementaria a la de la competencia entre las empresas españolas del alambre, pero se destacan los más relevantes. En primer lugar, el escaso número de empresas importantes en el sector. En segundo, la permisividad del Estado ante la formación de cárteles y acuerdos colusivos que reducían la competencia y aumentaban los precios en el mercado español de productos de la trefilería. En tercer lugar, la voluntad de las empresas de mantener el control familiar de la propiedad y la gestión, evitando la injerencia de propietarios ajenos a la familia ${ }^{5}$. Por último, las relaciones, en general, próximas y fluidas, entre las principales empresas del sector y los representantes de las instituciones políticas (regionales y estatales), que facilitaron - como en otros sectores relativamente intensivos en capital- la mencionada permisividad ${ }^{6}$.

Este trabajo utiliza básicamente fuentes de archivos empresariales y archivos familiares privados y, en particular, la documentación de dos de las cuatro empresas españolas más importantes del sector en el período de referencia: la catalana Rivière y la asturiana Moreda, en activo desde 1854 y 1879, respectivamente - compradas entre 1980 y 1999 por Global Steel Wire- ${ }^{7}$. También se utiliza información publicada sobre la cántabra Quijano.

La investigación se centra en el período 1880-1974. En estos años se promulgaron leyes (fiscales, comerciales e industriales) que pretendieron proteger de la competencia y reducir la carga fiscal a los grandes grupos de manufacturas del alambre. Amparados en un marco institucional favorable y en un mercado caracterizado (excepto en los años de guerra y autarquía) por una demanda interindustrial expansiva, los acuerdos de cooperación entre grandes empresas del sector fueron frecuentes, y tuvieron como objetivo general mantener su control sobre el mercado

4 No obstante, Cabrera y Del Rey (2002) ofrecen una visión crítica de la idea de que las élites locales y regionales influyeron de manera determinante en la política española. Aunque estos autores defiendan la autonomía de la acción política en relación con los intereses económicos, lo cierto es que la política económica nunca es neutra y, en economía, "no intervenir" es, de hecho, dejar que intervengan los intereses de los grupos empresariales por encima de los de los consumidores, lo cual es intervenir.

La aversión a propietarios ajenos a la familia y, en particular, a ceder control a extranjeros, es una característica todavía muy marcada de las empresas familiares españolas del sector industrial, según Galve y Salas (2003).

6 Sánchez Recio y Tascón (2003).

7 Para una aproximación a largo plazo de ambas, Fernández Pérez (2004) y (en prensa). 
interior y evitar el descenso de los precios, al menos hasta la crisis de 1970. Además, según testimonios directos de empresarios, dichos acuerdos también pretendieron contribuir al mantenimiento de la estructura familiar de la propiedad de las empresas del sector, evitando injerencias externas (que una gran complejidad organizativa y escala productiva podrían haber facilitado en el caso de existir competencia real) y asegurando así la supervivencia del grupo familiar que controlaba cada empresa.

\section{El proceso de concentración en el sector del alambre de hierro y acero durante el siglo $\mathrm{XX}$}

Aunque existen trabajos sobre la concentración y la cartelización en algunos sectores capital-intensivos de la industria española - como los energéticos, el cemento y la siderurgia, entre otros-, apenas hay estudios, al respecto, relativos a industrias ligeras del metal ${ }^{8}$. La manufactura de alambre de hierro y acero es una de esas industrias, para la cual, no obstante, ya existe un número significativo de contribuciones ${ }^{9}$.

Antes de la Guerra Civil, el mercado del alambre en España estaba en expansión, según revela el notable crecimiento registrado por la importación de hilo metálico: 700 toneladas en 1854, 7.000 en 1900 y 16.000 en $1934^{10}$. Antes también de 1936, se había operado ya una progresiva sustitución de importaciones de manufacturas de alambre, gracias a un centenar y pico de establecimientos localizados cerca de núcleos urbanos de todo el país. Así lo apuntan los datos de producción agregados, si bien, con cierto rigor, sólo disponemos de los proporcionados por el Servicio de Estudios de Francesc Cambó para el año 1921: 12.000 toneladas para la provincia de Barcelona y unas 17.200 para el conjunto de España. El número de obreros de la trefilería sólo aparece desagregado, en la misma fuente, para el caso de la provincia de Barcelona, unos 1.000 en 1921. Si fuera cierto que Barcelona representaba cerca de la mitad de la producción del sector en España en dicho año, sería plausible estimar alrededor de 2.000 el número de obreros del alambre existente por entonces en el país ${ }^{11}$.

8 Sobre la cartelización en la distribución de hierros comerciales, Martínez Ruiz (1998); en la siderurgia, Fraile (1991); Alonso, Erro y Arana (1998); Ojeda (2000); Sáez García (2005), y González (2004); para otros sectores, véanse Comín y Martín Aceña (1996), y Valdaliso (1999).

9 Rivière (1954); Bustamante (1986); Puigdomènec (1993), Fernández Pérez (1999), (2004), y (en prensa), y Fábrica Siderúrgica Moreda (1954).

10 Fernández Pérez (2005).

11 Archivo Histórico de Francesc Cambó, Servicio de Estudios, Carpeta “Transformados Metálicos, 19211923". Los datos de la fuente son para el año 1921. 
Según datos procedentes de los archivos de la empresa catalana Rivière y de la cántabra Quijano, las condiciones de entrada al sector en la segunda mitad del siglo XIX no eran particularmente onerosas, pero sí requerían capacidad de conexión estable con proveedores, extranjeros y nacionales, y con artesanos cualificados del metal locales; $y$, además, habilidad para consolidar como clientes a grandes almacenistas y ferreteros de provincias. Es decir, precisaban que el empresario fuera capaz de convertirse en nodo estable de una amplia red local, nacional e internacional ${ }^{12}$. Gracias a la Estadística Administrativa de la Contribución Industrial de 1900 y al Anuario de la Unión Industrial Metalúrgica de 1935, sabemos que el número de talleres y fábricas del sector se situaba entre 140 y 200 en el primer tercio del siglo XX. Se trataba, por tanto, de un subsector pequeño, con pocos artesanos y fabricantes en comparación con otros subsectores artesanales o industriales del metal, como las herrerías o las fundiciones. Antes de la Guerra Civil, las empresas más importantes de la trefilería en España eran, en Cataluña, las de las familias Detouche, Olivella, Marull, Rosés, Rivière y la Société de Tréfilérie Franco-Espagnole; en el País Vasco, las de Perot, Alambres del Cadagua, Castrejana y Echevarría; en Cantabria, la de la familia Quijano; y en Asturias, la fábrica de Moreda ${ }^{13}$.

Después de la Guerra Civil y durante la segunda mitad del siglo XX, el sector se caracterizó por la tendencia a la reducción del número de empresas y centros productivos, así como por la concentración de la producción en un menor número de fábricas, siendo escasa la tendencia exportadora antes de la crisis de los setenta. No tenemos datos para todas las empresas del sector justo antes de 1974, pero disponemos de un estudio algo posterior, para 1988. La información que incluye no es la ideal para advertir la estructura empresarial del sector antes de los problemas de la década de 1970, pero es la única disponible más próxima a ese momento.

Teniendo en cuenta este problema, el contraste entre el primer tercio del siglo y 1988 revela una drástica reducción en el número de centros productivos en España y un aumento de la concentración de la producción en unas pocas empresas (Cuadro 1). En 1988, sólo constaba la existencia de 54 centros, que empleaban a 4.150 personas y producían 578.000 toneladas agregadas de manufacturas de alambre. Comparado con los cerca de 200 centros productivos, 2.000 plausibles empleados y 17.000 toneladas de principios de la década de 1920, se observa un gran crecimiento de la producción, una gran concentración del empleo en pocos establecimientos fabriles y un espectacular aumento de la productividad, así como un mayor peso de la exportación que de la importación en el comercio exterior del sector.

12 En la Estadística Administrativa de la Contribución Industrial de 1900 [Dirección General de Contribuciones (1901)], figuraban cerca de 12.823 herreros y cerrajeros (Sección "Artes y Oficios", p. 548). Sobre Rivière, Fernández Pérez (2004), y sobre Quijano, Bustamante (1986).

13 Dirección General de Contribuciones (1901), y La Industria Metalúrgica, 1922-1934. 


\section{CUADRO 1}

EMPRESAS DE LA TREFILERÍA EN EUROPA OCCIDENTAL, 1988

\begin{tabular}{lccccc}
\hline & $\begin{array}{c}\text { Producción } \\
\text { (miles Tm) }\end{array}$ & $\begin{array}{c}\text { Centros } \\
\text { productivos }\end{array}$ & $\begin{array}{c}\text { Número de } \\
\text { empleados }\end{array}$ & $\begin{array}{c}\text { Exportación } \\
\text { (Tm) }\end{array}$ & $\begin{array}{c}\text { Importación } \\
\text { (Tm) }\end{array}$ \\
\hline UE-15 & 5.051 & 312 & 29.550 & & \\
Alemania & 992 & 85 & 10.500 & 700 & 603 \\
Italia & 880 & 84 & 5.000 & 370 & 177 \\
Reino Unido & 954 & 25 & 3.400 & 198 & 202 \\
Benelux & 879 & 20 & 9.200 & 629 & 410 \\
Francia & 768 & 44 & 6.300 & 311 & 600 \\
España & 578 & 54 & 4.150 & 126 & 70 \\
\hline
\end{tabular}

Fuentes: Eurostrategy Consultants (1995) y datos manuscritos de Benjamín lbáñez, Secretario de la Asociación de Trefiladores del Acero de España. Ibáñez proporcionó una copia íntegra del informe, y corrigió las cifras originales que figuraban para España (31 centros productivos, 2.800 empleados, $171 \mathrm{Tm}$ exportadas).

Del Cuadro 1 se deduce que el tamaño promedio de los centros españoles se parecía, hace dos décadas, al de los italianos, pero era mucho menor que el del resto de países. También se aprecia que, en España, el grueso de la producción se destinaba al consumo nacional y no a la exportación, como ocurría en otros de los países considerados. Los contrastes en el tamaño de los centros productivos, en la productividad por empleado y en la capacidad exportadora, tienen mucho que ver con la distinta cronología de la innovación y del crecimiento del sector en Europa occidental.

Lo que nos interesa ahora, más que subrayar esta distinta trayectoria en términos comparados, es resaltar, de nuevo, que ya antes de la Guerra Civil el sector del alambre en España lo controlaba un número de talleres y empresas relativamente reducido, número que, durante la segunda mitad del siglo XX (como en otros países de Europa occidental para los que contamos con datos), se redujo aún más, a la par que aumentaba fuertemente su plantilla y su producción. Sin duda, la crisis de los setenta agudizó esa tendencia. Estas características de la estructura empresarial del sector del alambre constituirían un primer factor que, con toda probabilidad, facilitó, sobre todo en coyunturas de mercado e institucionales adversas, la articulación de intereses y la formación de entramados y redes cooperativas interempresariales ${ }^{14}$.

14 Aunque no conforman la única posibilidad de una mayor labor de presión ante instancias políticas, sí tienen importancia, como explica Callejón (2001), p. 143. 
La documentación conservada revela que la cooperación interempresarial y con las instituciones políticas posibilitó que las empresas del alambre con mayor cuota de mercado regional y nacional (Quijano, Moreda, Rivière) fueran las que mantuviesen su independencia como empresas familiares, con escasas injerencias extrañas, en el transcurso del siglo ${ }^{15}$. Su tamaño (entre 200 y 1.000 empleados hasta la crisis energética), su escaso número y su concentración en algunas regiones de la periferia peninsular, favorecieron su influencia y capacidad de presión ante el Estado para obtener una legislación protectora de la competencia. También la formación de acuerdos de cooperación interempresariales. Las redes personales, familiares, regionales y de conveniencia económica fueron eficientes y funcionales en la industria del alambre española porque el marco institucional en que se desarrollaron no fijó, ni mantuvo, reglas estables de funcionamiento del mercado. Y cuando las fijó de forma coyuntural, fue para reducir el impacto de la competencia exterior, en contraste con otros países, en los que fue el poder político el que canalizó y estimuló una mayor competitividad exterior de las empresas de la trefilería $^{16}$.

Las empresas españolas del alambre de hierro y acero más importantes ejemplifican, por tanto, lo que las teorías sociológicas y de la dependencia indican sobre la relevancia que tienen los entornos personalistas y arbitrarios en la definición, también personalista y arbitraria, de las leyes de propiedad y de la competencia. También demuestran que la relativa protección estatal y la colusión empresarial permitieron, en esta industria, una relativa sustitución de importaciones y el desarrollo de un sector industrial auxiliar de numerosas actividades económicas. El coste de esa sustitución, al elevarse las barreras de entrada para potenciales competidores y los precios para los consumidores españoles, y al prolongar el atraso tecnológico español, es un tema que, pese a su importancia, no se trata en este estudio.

A continuación, se presentan algunas de las coyunturas en las que mejor se pueden visualizar los acuerdos de cooperación interempresarial en las empresas del alambre, y los elementos más relevantes que caracterizaron dichos acuerdos. Las industrias del alambre emplean metales muy distintos con diferentes implicaciones, por su tecnología y sus mercados. En esta ocasión, sólo nos referiremos

15 El caso mejor documentado es el de los Rivière que, acogiéndose a la legislación mercantil vigente, realizaron dos acuerdos de sindicación de acciones en 1935 y 1955, bloqueando la salida de acciones con derecho a voto de la empresa entre 1935 y 1961, para evitar — aún siendo anónima— la cesión de control a favor de personas o entidades ajenas a la familia. La sindicación preveía opciones preferentes de compra de tales acciones para los primitivos accionistas antes de ofertarlas en el mercado libre. Archivo Histórico de Francisco Rivière Ribas, Carpeta 5, Rivière S. A., y Fernández Pérez (2004), p. 153. 
a las que utilizaron principalmente hierro y acero. Por otra parte, debido a la falta de información disponible, no se tratarán aspectos relativos a la red de distribución comercial de las empresas de trefilería, pese a su importancia para explicar el mantenimiento del personalismo y el capitalismo familiar en el sector. Durante el siglo XX, la trefilería en España ha manufacturado una enorme diversidad de productos hechos con alambre, cuya distribución ha supuesto altos costes de transacción e información por su transporte (son productos de elevado peso y valor unitario relativamente bajo) y por el elevado personalismo existente en la distribución comercial. Según las empresas Quijano, Moreda, Echevarría y Rivière, los precios de los productos dependían no sólo de variables económicas, sino también de quién fuese el cliente y de la negociación personal efectuada por los viajantes y representantes de las empresas, que solían tener margen de maniobra respecto del establecimiento de precios con autorización de los fabricantes ${ }^{17}$. La elevada diversidad de la clientela y las deficientes infraestructuras del país hicieron que la articulación de redes comerciales en España fuese compleja y personalizada hasta la década de 1970. En el caso de la trefilería, y según un informe de principios del decenio de 1960, hecho por consultores belgas pagados por la multinacional Bekaert, para un mismo artículo de alambre, como el mallazo, podían encontrarse en el mercado español hasta tres o más precios distintos ${ }^{18}$. La complejidad en la fijación de precios (no sólo según las fluctuaciones de los mercados de materias primas y productos acabados, sino también según el cliente) y en la distribución de productos de alambre en España, hizo que esta multinacional firmase un acuerdo de cooperación técnica y de financiación con la empresa Rivière, a cambio de usar las redes comerciales de la misma e introducir, así, sus productos en el mercado español ${ }^{19}$. La capacidad de articular redes comerciales personales estables y complejas fue, por tanto, un factor que desempeñó, sin duda, un papel importante en la larga supervivencia de aquellas empresas que, como Rivière, fueron capaces de captar y fidelizar a grandes clientes regionales redistribuidores durante generaciones $^{20}$. Sin embargo, no tenemos datos similares para otras empresas del sector que lo confirmen y, sobre todo, no disponemos de documentación procedente de grandes ferreteros y almacenistas que corroboren con mayor detalle esta apreciación.

Archivo Histórico de Moreda Rivière Trefilerías S. A., Carpeta que contiene Historial de la empresa, y Reconversión Industrial y Acción Concertada, septiembre 1978.

18 Archivo Histórico de Francisco Rivière Ribas, Carpeta Bekaert, Documentos de la $1^{a}$ entrevista con los Sres. W. y J., 28-4-1960.

19 Archivo Histórico de Francisco Rivière Ribas, Carpeta Bekaert, Documentos de la $1^{a}$ entrevista con los Sres. W. y J., 28-4-1960.

20 


\section{Empresarios, coyunturas de mercado y redes asociativas (finales del siglo XIX y primer tercio del XX)}

En España, por lo menos desde el primer tercio del siglo XIX, se habían venido importando manufacturas, como telas metálicas, hilos de alambre de varios diámetros y enrejados, desde Francia, Inglaterra y Alemania. A finales del siglo XIX, los artículos importados habían permitido conocer la tecnología (relativamente simple) empleada fuera de nuestras fronteras en el sector del alambre, mediante su instalación, mantenimiento y reparación. El aprendizaje práctico había facilitado la transferencia tecnológica a trabajadores con escasa experiencia en el sector industrial ${ }^{21}$. Y también, hasta la década de 1880, a capitalistas y técnicos interesados en crear empresas en un sector que entonces tenía costes de entrada relativamente bajos, y donde los principales activos consistían en el contacto con proveedores de dentro y fuera de España, y en habilidades comerciales para conquistar los mercados regionales españoles.

Algunos de estos empresarios eran de origen francés, como François Rivière Bonneton, o José Tartière e Isidoro Claussel (de Trefilería Moreda), pero hubo destacados autóctonos, como el cántabro José María Quijano. La vinculación a intereses franceses, ferroviarios (Rivière) y financieros (Claussel, Tartière), así como la asistencia a exposiciones comerciales en Francia y España (Rivière a la de Agricultura de Madrid de 1857, Quijano a la de París de 1873), fueron el marco de acercamiento apropiado a tecnología y empresas innovadoras, y en su seno se articularon nuevas redes de intereses y de transferencia de información y contactos.

A partir de esta red, establecida con un entorno innovador, se activó otra, de parentesco y/o paisanaje de cada emprendedor, para reunir recursos que financiasen o apoyasen con factor trabajo una aventura empresarial en un sector en el que no había experiencia previa (Rivière era contratista de obras de ferrocarriles y dibujante, Quijano abogado, Claussel ingeniero) ${ }^{22}$. Las redes de parentesco y paisanaje fueron fundamentales para que Rivière y Quijano tuvieran sus primeras - pequeñas, pero muy leales- plantillas de personal, técnicos y directivos. Estos primeros técnicos, parientes o paisanos, ejercieron a su vez de nodos que articulaban redes de reclutamiento y formación (in-house training), en la propia región (Quijano, Rivière),

\footnotetext{
21 Los registros de personal de tres fábricas de Rivière recogen datos sobre el origen del personal y su experiencia previa. Hacia 1900, un elevado porcentaje procedía de Castellón, Murcia y Tarragona, zonas sin tradición industrial (aunque sí minera, en algunos distritos,). Archivo Moreda Rivière Trefilerías S. A., Registros de Personal (1871-1941). Una elaboración de estos datos en Fernández Pérez (2004).

22 Quijano no tenía suficientes recursos propios. Logró la ayuda financiera de su acaudalada suegra, su prima hermana, su tío Benigno de Arce y su amigo Pedro Ruiz Tagle. Bustamante (1986), pp. 142 y 147.
} 
y en regiones próximas (Rivière) ${ }^{23}$. Así, gracias al consejo de un sacerdote, asiduo visitante de la casa de su suegra, José María Quijano, un abogado sin experiencia industrial, recibió la recomendación de emplear a Domingo Salas Cianca, herrero y cerrajero de Zurita, como hombre de confianza que podía organizar la nueva fábrica de puntas de Quijano en Los Corrales de Buelna. La relación entre la familia de Salas y los Quijano duraría generaciones, y aseguró el apoyo técnico directo del nuevo negocio. Los buenos sueldos pagados por Quijano sellaron con estímulos concretos las promesas y la confianza mutua y atrajeron a otros herreros, campesinos y ganaderos modestos del valle de Buelna ${ }^{24}$. En el caso de François Rivière, inicialmente (1860-1883) la relativa falta de contactos en un país que no era el suyo y la movilidad desplegada en busca de una localización óptima para taller y almacenes (Madrid, Bilbao, Valladolid, Barcelona), hicieron que le faltase el entramado de contactos regionales de que disfrutó, desde sus inicios, la empresa de Quijano en Cantabria. François Rivière Bonneton precisó más tiempo para construir dicho entramado y, hasta que lo tuvo (en Cataluña, a partir de la década de 1880), le ayudó en la dirección y en sus intentos de consolidarse en Madrid, y asentarse en Bilbao y en Valladolid, un familiar próximo: su hermano Antoine. Éste aprendió de François en el taller de Madrid cómo llevar los telares y qué problemas técnicos surgían al fabricar distintos tipos de hilos de alambre. Partiendo de este aprendizaje, pudo supervisar la contratación y las labores realizadas por el personal del taller instalado en Bilbao (1868-1874). Una iniciativa individual de Antoine en la década de 1880 quebró la confianza que su hermano le había venido otorgando. A partir de entonces fue cuando François Rivière amplió sus relaciones con la élite de negocios barcelonesa, instalándose en pleno Paseo de Gracia, haciéndose socio del Fomento del Trabajo Nacional, participando en la redacción de informes impulsados por esta patronal catalana contra el proyecto de reforma arancelaria de Moret, y vinculándose mediante lazos de parentesco (los matrimonios de sus hijos) con familias destacadas de papeleros (Torras), industriales textiles (Manén, Massana) y del cáñamo (Caralt) ${ }^{25}$.

En un proceso paralelo a la consolidación de estas empresas con instalaciones, maquinaria y personal apropiados, se organizó la distribución comercial. Aunque el mercado interior fue siempre el principal — de mediados del siglo XIX a la década de 1980-, hubo dos momentos en que las pequeñas empresas españolas del alambre exportaron: antes de la pérdida de las últimas colonias de Ultramar y en

23 Sobre Rivière y Tartière, Fernández Pérez (1999) y (2004); Rivière (1954); Cabana (1992), pp. 167-170; y Fábrica Siderúrgica Moreda (1954). Sobre Quijano, Bustamante (1986); Trefilerías Quijano (1998); y Laguillo (2001).

24 Bustamante (1986).

25 Fernández Pérez (2004). 
la primera mitad de la década de 1930. Cabe advertir que se trataba de cantidades muy pequeñas, enviadas a mercados cautivos o afines: en la década de 1880, la empresa Rivière exportaba cerca de 350 toneladas de sus manufacturas a Cuba y Puerto Rico ${ }^{26}$. Hacia 1934, todo el sector exportaba sólo unas 2.600 toneladas, cuyo valor era unas cien veces inferior al de la importación de productos de alambre de dicho año $0^{27}$. Su dimensión era demasiado reducida para garantizar la viabilidad de las empresas españolas del sector. La competencia de las empresas alemanas, belgas, francesas e italianas, tecnológicamente más avanzadas, complicaba la salida al exterior ${ }^{28}$. El mercado del que dependían estas pequeñas empresas era el interior.

Pero en la década de 1890, las posibilidades de desarrollo en el mercado español de las que serían en el siglo XX grandes empresas del alambre (Rivière, fundada en 1854/1860, Quijano, en 1873, Moreda, en 1879) no estaban claras: la política comercial norteamericana situó en su punto de mira los mercados antillanos, que España acabó perdiendo, y, sobre todo, la crisis de precios de finales del siglo XIX perjudicó gravemente a los principales clientes (ferreteros distribuidores en regiones rurales, explotaciones mineras y empresas agroalimentarias) del propio mercado peninsular. Ante la grave crisis de demanda, los empresarios del norte de España iniciaron un proceso de asociación y cooperación a escala regional que llevó, en los años del cambio de siglo, a la creación de la Central de Fabricantes de Alambres y Puntas (por empresas cántabras, asturianas y vascas) y de la Unión de Fabricantes de Alambres y Puntas de París (por empresas catalanas). Este asociacionismo era paralelo al que se estaba registrando entre los empresarios siderúrgicos, cuyos acuerdos influyeron en una tendencia al alza de los precios de los productos consumidos por los transformadores de metal — como los fabricantes de alambre y sus derivados- ${ }^{29}$. Entre los motivos de la existencia de dichas asociaciones, los documentos consultados indican el de estudiar el impacto de la política comercial de otros países y los efectos de las medidas de política arancelaria tomadas por los gobiernos españoles. E investigar, también, las consecuencias del encarecimiento de los productos siderúrgicos y de la progresiva integración de transformados por parte de empresas siderúrgicas del norte, que podía reducir el consumo de materias intermedias de los transformadores de metal del resto de España ${ }^{30}$. Es decir, los empresarios del alambre se unían en

\footnotetext{
26 Fernández Pérez (2004).

27 Ministerio de Hacienda (1934). Era una exportación más que relativa: un 65 por 100 del total iba a las posesiones españolas en el norte de África y a Canarias, y del resto, el 15 por 100 se dirigía a algunos países latinoamericanos.

Sobre las italianas, en el contexto europeo, Colli (1999).

Sáez García (2005).

Archivo Histórico de Francesc Cambó, Servicio de Estudios, Carpeta Transformados metálicos, 1921-1923.
} 
asociaciones para aminorar costes de información. Y para reducir costes de transacción, mediante la presión de sus lobbies en Madrid; se organizó un selecto grupo de empresarios representante de cada región, que dialogó con asociaciones de otras regiones para hacer propuestas comunes frente a los temidos aumentos de los costes de importación de materias primas y maquinaria. Las memorias de las Juntas de socios del Fomento del Trabajo Nacional, y de su Agrupación de Industrias Siderúrgicas y Metalúrgicas, dan fiel testimonio de la vitalidad del movimiento asociativo barcelonés y de sus actividades, tanto elaborando informes y estadísticas como viajando a Madrid para entrevistarse con los responsables de la política comercial e industrial durante las décadas de 1890 y $1910^{31}$. Una de las escasas biografías sobre el fundador de Nueva Montaña Quijano, portavoz de los industriales del alambre vascos, cántabros y asturianos (hasta su fallecimiento en 1911), revela que estos últimos desarrollaron su presión sobre los políticos de Madrid de manera más directa que los catalanes, gracias a la disponibilidad de redes de amistad y paisanaje con destacados políticos de origen cántabro y vasco residentes en la capital. Uno de los casos más notables fue el de la relación personal entre la familia Quijano y Antonio Maura: Gilberto Quijano, hermano de José María Quijano, llegó a compartir piso en Madrid, en la calle de Alcalá, con Antonio Maura y con el senador montañés Leandro de Alvear, y fue diputado por Puerto Rico. Maura fue amigo personal de los Quijano toda su vida ${ }^{32}$.

No tenemos documentos que indiquen de forma directa el impacto y la funcionalidad de estas redes en orden a lograr los objetivos perseguidos por las mencionadas asociaciones de empresarios del alambre. Pero sí existen evidencias indirectas sobre la receptividad del Estado a algunas de estas presiones y el efecto beneficioso de las medidas gubernamentales en la marcha de algunas empresas catalanas. Así ocurrió con la revisión de algunas de las tarifas arancelarias que habían estado vigentes desde 1869, de manera que el promedio de las que gravaban los alambres de hierro, muelles y redes de alambre importados aumentó del

31 Archivo del Fomento del Trabajo Nacional, Francisco Rivière, Telas metálicas, en Contestación al Voto Particular del Excmo. Sr. Don Segismundo Moret Prendergast al Dictamen de la Comisión Arancelaria, Barcelona, Tipografía Hispano-Americana, 1891, pp. 31-50. Memoria leída en la Junta General Ordinaria de socios celebrada el día 29 de enero de 1893, Barcelona, Imprenta de Henrich y Cía, 1893. Memoria leída en la Junta General Ordinaria de socios celebrada el día 26 de enero de 1896, Barcelona, Tipografía Española, 1896. Memoria leída en la Junta General Ordinaria de socios de 31 de enero de 1904, Barcelona, Imprenta de Domingo Casanovas, 1904. Agrupación de Industrias Siderúrgicas y Metalúrgicas del Fomento del Trabajo Nacional, Memoria de los trabajos realizados hasta la fecha por la actual Junta Directiva leída en la Junta General celebrada el 13 de mayo de 1909. Exposición Internacional, Asesoría Informativa-Organización de Propaganda, Índice y clasificación de las industrias correspondientes a cada una de las Provincias de España, 1915.

32 Bustamante (1986), pp. 228, 233, 235, y 238.

33 Ministerio de Hacienda (1855) y (1902). 
20-25 por 100 de 1854 a cerca del 40 por 100 en $1900^{33}$. Este alza de los aranceles benefició directamente a los productores españoles de manufacturas del alambre, según confirman testimonios del propio François Rivière Bonneton. Éste había denunciado en 1891 la importación masiva de telas metálicas y alambres extranjeros con escasa carga impositiva en el apartado que, sobre Telas Metálicas había escrito en la Contestación al voto particular del Excmo. Sr. D. Segismundo Moret y Prendergast al Dictamen de la Comisión Arancelaria. Sin embargo, en 1905, afirmaba en una nota manuscrita que, por entonces, ya no entraban prácticamente en España telas metálicas como las que antes, habitualmente, llegaban del exterior: "hoy no entran ya telas del extranjero, tan sólo las que entran bajo declaraciones falsas, y que (...) fabrica [la casa Rivière] todas las clases de telas desde las mallas de $70 \mathrm{~mm}$., hasta la malla de 0,05 mm., telas para fábricas de papel sin fin hasta el número $250^{\prime \prime 34}$. Estaba claro que la protección había ayudado a reducir la importación de las manufacturas metálicas que estaban produciendo las "grandes" empresas españolas del alambre desde finales del siglo XIX.

Además de la protección, el avance tecnológico, la flexibilidad del mercado laboral y el aumento de las inversiones que se registraron en algunas fábricas españolas del alambre, fueron factores que facilitaron la citada sustitución de importaciones. Aprovechando el paraguas protector y el impulso de la demanda debido a la I Guerra Mundial, primero, la política de infraestructuras de la Dictadura de Primo de Rivera y la expansión de la demanda interindustrial, después, algunas de las principales empresas del país, como Quijano, Moreda y Rivière, incrementaron sus inversiones para eliminar competidores locales, a través de la compra de empresas pequeñas de sus regiones respectivas, algunas de ellas en quiebra, durante el primer tercio del siglo XX. Redujeron, así, el número de competidores y de centros productivos en cada región, algo que, aunque es difícil de precisar de modo cuantitativo, cualitativamente confirman los documentos consultados de las principales empresas del sector $^{35}$. Dichas empresas habían ampliado su capacidad productiva y habían acelerado su mecanización, si bien los mercados exteriores logrados en la coyuntura de la I Guerra Mundial, aunque importantes, resultaron efímeros. En el decenio de 1920, quedaron limitadas, de nuevo, a dirigir su producción al mercado interior, y a unos precios en descenso como efecto del aumento de la productividad y la capacidad productiva.

34 Archivo Histórico del Fomento del Trabajo Nacional, Contestación al voto particular del Excmo. Sr. D. Segismundo Moret y Prendergast al dictamen de la Comisión Arancelaria, Barcelona, Tipografía Hispano-Americana, 1891, pp. 31-50. La cita de 1905 pertenece a una breve nota escrita por Francisco Rivière Bonneton titulada Historique de la Maison, en Archivo Histórico de Francisco Rivière Ribas.

35 Si se ha estimado para Cataluña en 1920-1922; véase Calvo (1985). 
Como en otros sectores y países - y como indica la economía industrial (Callejón, 2001) - la concentración de la producción y la eliminación de competidores facilitaron la colusión empresarial. Además, como los cárteles no estaban prohibidos por ley y el Estado no impedía los acuerdos restrictivos de la competencia, las principales empresas asturianas, cántabras, vascas y catalanas acordaron cuotas de producción por artículos y asignaciones de mercados regionales por productos. Como señala la teoría económica tradicional sobre cárteles - y el caso de la trefilería confirma lo demostrado recientemente por Sáez para la siderurgia española entre 1871 y 1907 (Sáez, 2005)—, el reparto del mercado y la detección de los transgresores eran los puntos clave de la existencia de un cártel. En 1920-1922, once empresas concentraban en Cataluña el 88 por 100 de las máquinas de trefilar y el 70 por 100 de los telares que tejían tela metálica. Por otra parte, y a escala nacional, la Central de Productores de Alambres y Puntas de Cantabria, Asturias y País Vasco, y la Unión de Fabricantes de Cataluña, creadas en la primera década de siglo, se unieron en un cártel en 1925: la Sociedad Anónima de Trefilería y Derivados, cuya escritura de constitución se firmó en San Sebastián en octubre de dicho año ${ }^{36}$. Las memorias que escribió para uso exclusivo de sus hijos el industrial Francisco Luis Rivière Manén, hijo y sobrino de los principales representantes catalanes en el cártel (Francisco y Fernando Rivière Chavany), proporcionan datos sobre las empresas que lo integraron, sus objetivos y los mecanismos utilizados para implementar la colusión ${ }^{37}$. Fueron diez las empresas integrantes, siete pertenecientes a la Central de Fabricantes de Alambres y Puntas, con sede en Madrid, (S.A. Quijano de Los Corrales de Buelna — que había comprado la catalana Marull en 1917—en la provincia de Santander; Alambres del Cadagua, Echevarría S.A., Trefilería Barbier y la Sociedad Franco-Española de Cables de Acero en Bilbao; Hijos de Mendizábal en Durango; y la Sociedad Industrial Asturiana-Moreda en Gijón); y tres pertenecientes a la Unión de Fabricantes catalana (Rivière, Metalúrgica Rosés y la fábrica de puntas Olivella, de Laureano Moreno Morcillo, casado con la hija de Olivella). Las fábricas del norte tenían producciones masivas de artículos de mayor contenido en carbono, y las fábricas catalanas una gran diversidad de derivados del alambre con alto contenido en trabajo. Se iniciaron conversaciones entre estos grupos de empresas hacia 1922, se realizaron ventas rápidas para aumentar la potencia de cada empresa en la negociación (quienes más vendían, más cuota podían obtener en el acuerdo) y, en tres años, se firmó el primer convenio relativo a alambres y puntas, creándose, como hemos indicado, el cártel español de la trefilería en 1925. En años sucesivos se firmarían otros convenios relativos a la amplia gama de derivados del alambre (especialmente telas, enrejados, espino y mallas).

36 Fernández Pérez (2004) p. 78. El dato sobre Cataluña procede de la tesis doctoral inédita de Calvo (1985).

37 Archivo Histórico de Francisco Rivière Ribas, Francisco Luis Rivière Manén, Memorias. Vol. I, pp. 126-132 (mecanografiado). 
El grupo de empresas del norte había integrado en sus fábricas la producción de fermachine o alambrón (materia prima para fabricar hilos de metal). En cambio, el grupo catalán apenas había comenzado a reducir su fuerte dependencia de aquél para la obtención de fermachine, pero tenía el apoyo estratégico de Altos Hornos de Vizcaya, que se lo suministraba para intentar frenar el crecimiento de Quijano, Moreda y Echevarría y su independencia respecto de la siderurgia bilbaína. En esta carrera por aumentar el aprovisionamiento de materia prima, a fin de incrementar la capacidad de producción de derivados, los precios siguieron una tendencia a la baja. La voluntad de permanecer independientes de las empresas familiares en competencia por el control del mercado interior, y de cerrar el paso a inversores ajenos a los grupos familiares, favoreció la búsqueda de acuerdos cooperativos para mantener precios e intentar controlar el avance de los rivales en mercados ocupados por productos de cada empresa y grupo regional. En los años veinte, cuando se estaban formando grandes uniones patronales del metal en Europa, intentar la conquista de los mercados exteriores por parte de las pequeñas empresas españolas — reacias a la entrada de capital extranjero- no era una alternativa viable ${ }^{38}$. En cambio, los acuerdos dentro de cada región eran factibles, por el escaso número de empresas y porque, a esa escala territorial, cada empresa solía poseer cierta especialización en una gama de artículos, lo que reducía los niveles de competencia. La auténtica competencia provenía, en esos momentos, de empresas ubicadas en otras regiones, que habían incrementado su capacidad transformadora y podían apropiarse de los clientes de los territorios tradicionales de cada una de ellas. La guerra de precios podía hundir a todas y, por ello, el cártel de 1925 jugó un papel importante - aunque breve, por el estallido de la Guerra Civil-, asegurando a cada empresa cuotas por productos en el mercado nacional. Confirmando las ideas de la teoría económica tradicional sobre cárteles, la existencia de unos líderes diferenciados en cada bloque regional (Quijano en el norte, Rivière en Cataluña) facilitó las negociaciones, de por sí duras, para acordar el reparto del mercado. Los catalanes pudieron garantizar su dominio en los derivados del alambre (telas metálicas y enrejados de triple torsión, por ejemplo) y los cántabros en la fabricación de alambre y cables (más intensiva en energía). Aunque la guerra y el franquismo alteraron la evolución del sector y las estrategias de cada empresa, lo cierto es que esta especialización regional por grandes líneas productivas se ha mantenido hasta fechas recientes ${ }^{39}$.

38 Archivo Histórico de Francesc Cambó, Servicio de Estudios, Carpeta Transformados metálicos, 1921-1923.
39 Fernández Pérez (2004). 


\section{La cooperación ante las rigideces y disfunciones del franquismo}

La Guerra Civil interrumpió estos acuerdos cooperativos, así como las redes comerciales y de aprovisionamiento de materias primas. El nuevo Estado impuso nuevas reglas de juego, caracterizadas por una intervención disfuncional ${ }^{40}$. Las principales empresas del sector del alambre sobrevivieron, con problemas, gracias a diversos factores, entre los que destacó inicialmente la excelente relación personal establecida entre algunos de los empresarios y los generales del ejército franquista, los jefes de la Falange y la nueva estructura de poder local que restituyó las empresas a sus antiguos dueños $^{41}$.

Durante la dictadura franquista, la creación del INI y la imposición de nuevas condiciones productivas significaron para las grandes empresas de la trefilería nuevos retos adaptativos, pero también nuevas oportunidades de negocio. La reducción de la transferencia tecnológica del exterior, por los obstáculos impuestos al comercio exterior por las autoridades franquistas y las restricciones a la propiedad extranjera de empresas españolas, junto con los efectos inflacionarios de la intervención en los precios, hicieron retroceder las cifras totales de producción de empresas como Rivière (que no recuperó los niveles de 1935 hasta 1953) y alteraron las estrategias de transferencia tecnológica e innovación previas a la guerra. Sin embargo, la introducción de jurados de empresa y la supresión de la libertad sindical aseguraron una relativa paz laboral y bajos costes salariales por trabajador hasta principios de la década de 1970. Además, la legislación que, a partir de 1939, reguló el número y tamaño de los centros productivos existentes en el país, favoreció que las grandes empresas siguieran controlando como antes de 1936 el mercado interior, permaneciendo a salvo de la competencia nacional y extranjera.

Las nuevas condiciones institucionales permitieron el continuado dominio de las mismas "grandes" empresas familiares después de 1939, las cuales se vieron beneficiadas, además, en reconocimiento del apoyo directo que algunos de sus miembros habían prestado durante la guerra a los militares sublevados (en el frente, en el servicio de espionaje, en las fábricas de material militar y en el servicio de propaganda). Asimismo, empresas del alambre, como Quijano, Moreda y Rivière, crecieron a partir de los años cincuenta por la expansión de la demanda interindustrial: filtros, enrejados y cables fueron crecientemente demandados por la construcción (mallazo, cables de acero) y la automoción (muelles para asientos y cables reforzados para los neumáticos de las ruedas), amén de la tradicional demanda del sector agrario. El marco regulador del franquismo, sin embargo, supuso rigideces para flexibilizar el aumento

$40 \quad$ Sánchez Recio y Tascón (2003).

41 Archivo de la Cámara de Comercio, Industria y Navegación, Comisión de Incorporación Industrial. 
de la capacidad productiva, necesario para responder al de la demanda potencial. Hasta finales de la década de 1950 hubo una gran escasez de materias primas y energía que perjudicó particularmente a la industria privada, así como una caída de la productividad laboral y de la disponibilidad de divisas ${ }^{42}$.

Esta nueva coyuntura crítica para el sector activó de nuevo, entre las grandes empresas supervivientes, el recurso a los acuerdos de cooperación. Los motivos de carácter económico explican buena parte de este nuevo movimiento asociativo entre las grandes empresas de la trefilería, y también el deseo de muchas de ellas de mantener su autonomía como empresas familiares y evitar la quiebra o la penetración de capital ajeno (español en todo caso porque, de 1939 a 1959, el capital extranjero tuvo reducida a mínimos su capacidad legal para participar en empresas españolas).

En el caso catalán, el esfuerzo, no siempre fácil para las partes implicadas, se dirigió a crear sociedades nuevas entre empresarios siderúrgicos y de los transformados ligeros, para asegurar el abastecimiento de carbón y alambrón. El libro publicado sobre Can Torras dels Ferros por Francesc Cabana y Assumpció Feliu, la obra conmemorativa de la historia de Roca Radiadores y las Memorias inéditas de Francisco Luis Rivière Manén ofrecen testimonios complementarios que informan sobre los esfuerzos realizados en Cataluña para contrarrestar la reducción de alambrón y acero que llegaban del norte antes de la guerra ${ }^{43}$. Una primera iniciativa, tomada por destacados empresarios catalanes del metal para, sin enfrentarse con el régimen franquista ni mucho menos, reducir los efectos negativos de su política económica en materia de costes productivos, fue Siderúrgica Asturiana S.A. (SIASA), constituida en Madrid en 1942. SIASA tuvo como objetivo producir hierro y acero de baja calidad, con la tecnología alemana Renn-Krupp, en las minas asturianas de Avilés, para enviarlo a las fábricas catalanas que habían invertido sus capitales en el proyecto. Estas empresas fueron Torras Herrería y Construcciones, Aceros Calibrados M. Aracil Bonas, A. Bianchini Ingenieros Trefilería, Tubos y Perfiles S.A., Metales y Platería Ribera S.A., Rivière S.A. y Altos Hornos de Cataluña (comprada por Rivière ese mismo año con idéntico objetivo: abastecerse de materia prima). El segundo esfuerzo colectivo de cooperación consistió en la adquisición hacia 1951-1953 de la antigua fábrica de Mieres (c. 1879), de nuevo en la zona carbonera de Asturias, por cuatro empresas catalanas: Rivière, Torras, Suñer-Comercial de Laminados y Roca. Ambas iniciativas tuvieron corta vida, por la intervención del sector público en el caso de SIASA, y por los duros conflictos internos entre las empresas catalanas en el caso de la fábrica de Mieres ${ }^{44}$.

42 Catalan (1995) y Sánchez Recio y Tascón (2003).

43 Luján (1992); Cabana y Feliu (1987); Fernández Pérez (2000).

44 Fernández Pérez (2004). 
Desde que empezaron a mejorar las condiciones que afectaban a la producción y a las ventas nacionales, desde mediados de la década de 1950, la colaboración entre las empresas del metal cambió sus formas y objetivos. Se hizo más visible la cooperación para lograr la innovación mediante la transferencia tecnológica desde el exterior, un proceso complicado en la España de la época que requería el esfuerzo organizado de empresas e instituciones ${ }^{45}$. Con este objetivo cabe entender buena parte de los viajes o "misiones" de la Comisión de Productividad Industrial (a empresas norteamericanas como IBM o Ford, y a grandes acerías y productores de alambre) y la creación de cursos de posgrado en dirección empresarial en nuevas escuelas privadas de negocios a finales de los años cincuenta, impulsadas por ingenieros industriales y empresarios ${ }^{46}$. También la formación de nuevas asociaciones entre empresas del metal alternativas al Sindicato Vertical, como el Centro de Estudios y Asesoramiento Metalúrgico (CEAM), creado en 1951, con fines de divulgación de novedades técnicas y asesoramiento, por impulso de tres destacados industriales catalanes del metal (Andrés Ribera Rovira, Miguel Elizalde Biada y Fernando del Pozo Querol) ${ }^{47}$. El deseo de informarse sobre el mercado exterior y la situación de la competencia internacional condujo a algunas empresas de trefilería a contactar con la American Steel Wire Association, fundada en 1930, y con asociaciones europeas de derivados del alambre como el Bureau Internationale des Toiles Métalliques (BITOM) creado en 1947 en Bruselas ${ }^{48}$.

Entre 1968 y 1974 la trefilería y los derivados experimentaron un fuerte crecimiento productivo en España. La transferencia tecnológica, por acuerdos de cooperación técnica o compra de licencias y patentes, y la inversión extranjera directa se incrementaron con rapidez en nuestro país. En algunas de las empresas líderes de la trefilería en España, como Rivière, se intensificaron los viajes al exterior (en 1957 a Estados Unidos, en una de las expediciones de las Comisiones de Productividad, y por Europa en las reuniones anuales del BITOM), se participó en las nuevas escuelas privadas de negocios (IESE) y en las nuevas asociaciones del metal (CEAM), y se dio entrada en el capital de la empresa a multinacionales (en 1961, Bekaert obtuvo 10 por 100 de Rivière a cambio de modernizar el proceso productivo y ampliar capacidad). También en estos años Quijano, Echevarría y Moreda incre-

45 Lo ha puesto de manifiesto recientemente la tesis de Mar Cebrián Technological Imitation and Economic Growth during the Golden Age, 1959-1973 (Instituto Universitario Europeo, Florencia, 2004). Agradezco a la autora que haya puesto a mi disposición un ejemplar de su trabajo.

46 Puig y Fernández (2003).

47 Fernández y Puig (2004) y Puig y Fernández (2003). Sobre el CEAM, Ortega y Hermosilla (2002).

48 Sólo Rivière, hacia 1949, y Codina, a principios de los años sesenta, habían sido miembros españoles de BITOM. Archivo Histórico de Francisco Rivière Ribas, BITOM, Rapport Confidentiel 1978-1979, Bruselas, 18 de mayo de 1979. 
mentaron su capacidad productiva, según se desprende de los datos internos de estas cuatro grandes empresas de poco antes de la crisis de 1974. Según estos datos, incluidos en la Acción Concertada de la Trefilería, el crecimiento de la producción española de alambres y sus manufacturas fue espectacular: de 17.200 toneladas agregadas de 1922 a 178.800 en 1973. Teniendo en cuenta que, según la información de estas empresas, la atonía productora dominó en la década de 1930 y que la producción de 1935 no se recuperó hasta 1953, las grandes empresas del alambre, a la altura de 1973, habían multiplicado por diez la producción española del sector en sólo veinte años. La modernización técnica y la casi inexistencia de competencia (debido a las rigideces institucionales) habían jugado un papel clave en este crecimiento, pero también otros factores: la permisividad y el apoyo institucional, el control del mercado por un reducido número de longevas empresas líderes, y la larga tradición de acuerdos cooperativos entre éstas, desde finales del siglo XIX hasta la Acción Concertada de $1974^{49}$.

\section{Conclusiones}

El desarrollo de la trefilería del hierro y el acero en España entre 1880 y 1974 se caracterizó por la concentración y la cartelización empresarial, no obstaculizada y a menudo favorecida por las políticas económicas de la década de 1890, de la Dictadura de Primo de Rivera, de la autarquía franquista y de los años de liberalización regulada de 1960-1974.

En el período estudiado, el Estado respetó la iniciativa de las grandes empresas privadas de la trefilería y dictó leyes que protegieron de la competencia y redujeron la carga fiscal a los grandes grupos de manufacturas del alambre. Amparados en un marco institucional favorable y con un mercado interior que, salvo en los años de guerra y autarquía, registró una demanda expansiva de productos industriales, los acuerdos de cooperación entre grandes empresas del sector abundaron.

El objetivo más directo de esta cooperación fue evitar caídas de precios y controlar el mercado nacional, por lo menos hasta la crisis de los años setenta del siglo XX. Además, y éste es un elemento poco o nada incluido en los estudios sobre cartelización y lobbies empresariales en España, la cooperación entre grandes empresas familiares tuvo también como objetivo evitar al máximo la entrada de socios ajenos al grupo familiar y/o regional que controlaba las empresas: tanto los Quijano en

Archivo Histórico de Francesc Cambó, Carpeta Transformados metálicos, 1921-23. Archivo Histórico de Francisco Rivière Ribas, Acción Concertada Siderurgia-Trefilería 1974-78. 
Cantabria, como los Tartière en Asturias o los Rivière en Cataluña estaban convencidos de que una competencia dura les forzaría a cambiar una estructura de la propiedad que ellos creían garantía de la supervivencia de su grupo familiar ${ }^{50}$.

Este trabajo indica otros factores que permiten entender la concentración empresarial y la frecuencia de acuerdos cooperativos en el sector. Entre ellos, el escaso número de empresas medianas y grandes en la trefilería del hierro y el acero en España, su concentración en unas pocas regiones (Cantabria, Asturias, País Vasco, Cataluña), la especialización en el mercado nacional, el apoyo de los centros de decisión políticos y un capital social acumulado durante generaciones, que facilitó la creación de redes entre empresarios y centros de poder (a escala local, regional y nacional), así como entre éstos y centros de innovación extranjeros. La integración de los empresarios de la trefilería en los círculos de sociabilidad de la élite de sus regiones respectivas facilitó el establecimiento de muchas de estas redes.

Los acuerdos cooperativos entre grandes empresas y empresarios españoles del alambre se realizaron históricamente hasta la década de 1920 a escala regional, para mantener el control sobre mercados regionales, particularmente tras la crisis de finales del siglo XIX. A partir de los años veinte, tuvieron progresivamente una dimensión nacional, en relación directa con el aumento de la competencia internacional y la tendencia de los precios, así como por la necesidad de hacer un frente común ante los aspectos más negativos del intervencionismo franquista en la organización de la producción, la distribución y la gestión. Acuerdos cooperativos entre empresarios españoles y extranjeros del sector se dieron con frecuencia en la segunda mitad del siglo XIX, se redujeron sensiblemente (aunque no dejaron de existir) durante la Guerra Civil y la autarquía, y se retomaron con brío en la década de 1960, y no sólo por razones técnicas o comerciales, sino también por estímulo de la ideología del catolicismo social que conectó patronales y empresarios europeos.

Cabría concluir que la cooperación interempresarial en este subsector dominado por pequeñas y medianas empresas familiares fue en el fondo, como han señalado en general Anna Grandori y Giuseppe Soda o Mark Granovetter y Mark Casson, una manera de regular la interdependencia entre empresas distinta $-\mathrm{y}$, a menudo, complementaria - a la de la integración vertical, y que se basó en unas reglas de

50 Este comportamiento no fue exclusivo de las empresas españolas, ni de las empresas del alambre, sino de numerosas empresas familiares enraizadas en economías regionales, como se pone de manifiesto en Colli, Fernández Pérez y Rose (2003). Como indicamos anteriormente, los Rivière hicieron dos acuerdos de sindicación de acciones con derecho de voto en 1935 y 1955 que evitaron la pérdida de control de la gestión familiar sobre el grupo empresarial Rivière hasta que se acordó, a cambio de transferencia tecnológica, dar un 10 por 100 de las acciones a un grupo belga en 1961. E, incluso en este ejemplo, se controló el grupo extranjero al que se permitía el acceso, ya que se seleccionó como socio a una empresa familiar centenaria, con cuyos miembros los Rivière habían tenido relaciones profesionales y de pertenencia a asociaciones patronales católicas europeas durante tres generaciones. 
juego no exclusivamente económicas, en las que intervinieron elementos de afinidad personal y regional ${ }^{51}$. La experiencia de las empresas del alambre fue en este sentido representativa, probablemente, de la de otras industrias dominadas por estructuras oligopolistas y familiares en las que existieron alternativas a la integración vertical, la estructura multidivisional y la gestión profesional de individuos ajenos a la propiedad empresarial.

\section{Fuentes}

Archivo Histórico de Francesc Cambó (Barcelona)

Servicio de Estudios, Carpeta Transformados Metálicos, 1921-1923 (1923).

Archivo Histórico de Moreda Rivière Trefilerías S.A. (Cerdanyola del Vallès).

Carpeta que contiene Historial de la empresa, Reconversión Industrial y Acción Con-

certada, Cerdanyola, septiembre 1978; y Registros de Personal, 1871-1941.

Archivo Histórico de Francisco Rivière Ribas (Barcelona)

Carpeta Bekaert. Documentos de la $1^{a}$ entrevista con los sres. W. Y J., Barcelona, 28-4-

1960; Carpeta 5 Rivière S.A.; Bureau Internacional des Toiles Métalliques (BITOM)

Rapport Confidentiel 1978-1979, Bruselas 18 mayo 1979; Acción Concertada Siderurgia-Trefilería 1974-1978 (mecanografiado); Historique de la Maison (s. d., mecanografiado); F. L. Rivière Manén, Memorias. Vol. I, pp. 126-132 (mecanografiado). Archivo del Fomento de Trabajo Nacional (Barcelona)

Francisco Rivière, Telas metálicas, en Contestación al Voto Particular del Excmo. Sr. Don Segismundo Moret Prendergast al Dictamen de la Comisión Arancelaria, Barcelona, Tipografía Hispano-Americana, 1891, pp. 31-50; Memoria leída en la Junta General Ordinaria de socios celebrada el dia 29 de enero de 1893. Barcelona, Imprenta de Henrich y Cia, 1893; Memoria leída en la Junta General Ordinaria de socios celebrada el día 26 de enero de 1896, Barcelona, Tipografía Española, 1896. Memoria leída en la Junta General Ordinaria de socios de 31 de enero de 1904, Barcelona, Imprenta de Domingo Casanovas, 1904; Agrupación de Industrias Siderúrgicas y Metalúrgicas del Fomento del Trabajo Nacional. Memoria de los trabajos realizados hasta la fecha por la actual Junta Directiva leída en la Junta General celebrada el 13 de mayo de 1909; Exposición Internacional. Asesoría Informativa-Organización de Propaganda, Índice y clasificación de las industrias correspondientes a cada una de las Provincias de España (1915). 
Archivo de la Cámara de Comercio, Industria y Navegación (Barcelona)

Comisión de Incorporación Industrial.

\section{Bibliografía}

ACTAS del X Simposio de Historia Económica, Análisis de Redes en Historia Económica, Bellaterra, 27-29 enero de 2005 (CD-ROM).

ALONSO, Eduardo J., ERRO, Carmen, y ARANA, Ignacio (1998): Santa Ana de Bolueta, 1841-1998, Bilbao, SPRI.

BEN-PORATH, Yoram (1980): “The F-Connection: Families, Friends and Firms and the Organization of Exchange", Population and Development Review, 6, pp. 1-30.

BORCH, Odd J., y ARTHUR, Michael B. (1995): "Strategic Networks among Small Firms: Implications for Strategy Research Methodology", Journal of Management Studies, vol. 32, pp. 419-437.

BUSTAMANTE, Ramón (1986): José María Quijano (Vida y obra de un hidalgo emprendedor), Santander, Nueva Montaña-Quijano.

CABANA, Francesc (1992): Empreses i empresaris. Els protagonistes de la revolució industrial a Catalunya, vol. Metal.lúrgics i químics, Barcelona, Enciclopedia Catalana.

CABANA, Francesc, y FELIU, Assumpció (1987): Can Torras dels Ferros 1865-1985. Siderúrgia $i$ construccions metal.liques a Catalunya, Barcelona, Tallers Gràfics Hostench.

CABRERA, Mercedes, y DEL REY, Fernando (2002): El poder de los empresarios. Politica y economía en la España contemporánea (1875-2000), Madrid, Taurus.

CALLEJÓN, María (2001): “Oligopolio", en CALLEJÓN, María (coord.), Economía Industrial, Madrid, Civitas Ediciones y Edicions de la Universitat Oberta de Catalunya, pp. 113-153.

CALVO, Ángel (1985): La transformación de la estructura industrial en Cataluña, 18981920, tesis doctoral inédita, Barcelona, Universitat de Barcelona.

CASSON, Mark (1999): "The Economics of the Family Firm", Scandinavian Economic History Review, 47, pp. 10-23.

CATALAN, Jordi (1995): La economía española y la segunda guerra mundial, Barcelona, Ariel.

CEBRIÁN, Mar (2004): Technological Imitation and Economic Growth during the Golden Age, 1959-1973, tesis doctoral inédita, Instituto Universitario de Florencia.

COHENDET, Patrick (ed.) (1998): The Economics of Networks: Interaction and Behaviours, Berlin, Springer-Verlag.

COLLI, Andrea (1999): Legami di ferro. Storia del distretto metallurgico e mecánico lecchese tra Otto e Novecento, Roma, Donzelli. 
COLLI, Andrea, FERNÁNDEZ PÉREZ, Paloma, y ROSE, Mary B. (2003): “National Determinants of Family Firm Development? Family Firms in Britain, Spain, and Italy in the Nineteenth and Twentieth Centuries", Enterprise \& Society, 4, pp. 20-64. COMÍN, Francisco, y MARTíN ACEÑA, Pablo (1996): "Rasgos históricos de las empresas en España. Un panorama", Revista de Economía Aplicada, 12, pp. 75-123. DIRECCIÓN GENERAL DE CONTRIBUCIONES (1857): Estadística Administrativa de la Contribución Industrial y de Comercio, 1856, Madrid, Impr. de L. García.

-(1901): Estadística Administrativa de la Contribución Industrial y de Comercio, 1900, Madrid, Impr. de L. García.

ECCLES, Robert G., y NORIA, Nitin (1992): Beyond the Hipe: Rediscovering the Essence of Management, Boston.

Estadística Histórica de la Contribución Industrial (1900).

EUROSTRATEGY CONSULTANTS (1995): The Community Wiredrawing Industry. Confidential Final Report to the European Commission. Directorate General III. Industrial Affairs, October 1995 (mecanografiado).

FÁBRICA SIDERÚRGICA MOREDA (1954): Fábrica Siderúrgica Moreda, 1879-1954, Bilbao, Editorial Vasca.

FERNÁNDEZ PÉREZ, Paloma (1999): “La empresa familiar y el síndrome de Buddenbrook en la España contemporánea. El caso Rivière, 1854-1979", en CARRERAS, Albert, PASCUAL, Pere, REHER, David, y SUDRIÀ, Carles (coords.), Doctor Nadal. La industrialització de l'Espanya contemporània, Barcelona, Publicacions U. B.

-(2000): “Leadership Succession in Spanish Family Firms, $19^{\text {th }}$ to $20^{\text {th }}$ Centuries", en KUIJLAARS, Anne-Marie, PRUDON, Kim, y VISSER, Joop (eds.), Business and Society, Rotterdam, Center of Business History, pp. 503-511.

-(2004): Un siglo y medio de trefilería en España. Moreda (1879-2004) y Rivière (18542004), Barcelona, Moreda Rivière Trefilerías S.A. (MRTSA) y Trivium.

-(2005): "Hilos de metal. La industria del alambre de hierro y acero en España (1854-1935)", Revista de Historia Industrial, 27, 1, pp. 165-192.

-(en prensa): "Francisco Rubiralta i Vilaseca y la creación de la primera gran multinacional catalana del metal: Compañia Española de Laminación S.A. (CELSA)", en CABANA, Francesc (dir.), Los cien empresarios catalanes del siglo XX, Barcelona.

FERNÁNDEZ PÉREZ, Paloma, y PUIG, Núria (2004): “Knowledge and Training in Family Firms of the European Periphery: Spain in the Eighteenth to Twentieth Centuries", Business History 46, 1, pp. 79-99.

FRAILE, Pedro (1991): Industrialización y grupos de presión, Madrid, Alianza.

GALVE GÓRRIZ, Carmen, y SALAS, Vicente (2003): La empresa familiar en España. Fundamentos económicos y resultados, Bilbao, Fundación BBVA.

GONZÁLEZ, Manuel Jesús (dir.) (2004): Hierro y acero ante la mundialización: una perspectiva histórica, Madrid, Aceralia-Grupo ARCELOR. 
GRANDORI, Anna, y SODA, Giuseppe (1995): “Inter-firm Networks: Antecedents, Mechanisms and Forms", Organization Studies, 16, pp. 183-214.

GRANOVETTER, Mark, y SWEDBERG, Richard (1992): The Sociology of Economic Life, Boulder, Westview Press.

LAGUILLO GARCÍA-BÁRCENA, Paulino (2001): Los Corrales de Buelna. Siglo XX (Resumen Histórico), Los Corrales de Buelna, Ayuntamiento de Los Corrales de Buelna.

La Industria Metalúrgica, revista de la Unión Industrial Metalúrgica, 1922-1934.

LUJÁN, Néstor (1992): “Historia de una familia de industriales", en LUJÁN, Néstor (ed.), La lucha contra el frío y el calor, y a favor de la higiene. Contribución de una familia de industriales catalanes a lo largo de 75 años, Barcelona, Montserrat Mateu Taller Editorial-Compañía Roca Radiadores S.A.

MARTÍNEZ RUIZ, José Ignacio (1998): La Unión de Almacenistas de Hierros y la distribución de hierros comerciales en España, Documento de trabajo de la Fundación Empresa Pública 9806, Madrid, Fundación Empresa Pública.

MINISTERIO DE HACIENDA (1855): Estadística General del Comercio Exterior de España, 1854, Madrid, Imprenta de Ramón Ballone.

—(1902): Estadística General del Comercio Exterior de España, 1900, Madrid, Establecimiento Tipográfico de J. Sastre y Compañía.

-(1934): Estadística General del Comercio Exterior de España, 1934, Madrid, Dirección General de Aduanas, Sucesores de F. Peña Cruz, Tomo 1.

OJEDA, Germán (2000): Duro Felguera. Historia de una gran empresa industrial, Oviedo, Grupo Duro-Felguera.

OLIVER, Amalya L. (1998): “Networking Network Studies", Organization Studies, 19, pp. 1-31.

ORTEGA GALÁN, Juan, y HERMOSILLA PÉREZ, Ángel (2002): Cincuenta años del CEAM y de la metalurgia catalana, Barcelona, CEAM.

PUIG, Núria, y FERNÁNDEZ, Paloma (2003): “The Education of Spanish Entrepreneurs and Managers: Madrid and Barcelona Business Schools, 1950-1975", Paedagogica Historica, 39, 5, pp. 651-672.

PUIGDOMÈNEC TEY, Francesc d'Assís (1993): Historial de la industria metal.lúrgica Rivière 1835, 1854-1979, La Garriga, Imprenta Tura Calàbria.

RIVIÈRE MANÉN, Francisco Luis (1954): Francisco Rivière, 1835-1911. Intimidades de una empresa barcelonesa, Barcelona, Limonograph.

ROBERTSON, Maxine, y otros (1996): "The Role of Networks in the Diffusion of Technological Innovation", Journal of Management Studies, 33, pp. 333-359.

ROSE, Mary B. (1999): "Networks, Values and Business: the Evolution of British Family Firms from the Eighteenth to the Twentieth Century", Entreprises et Historie, 22, pp. 16-20. 
SÁEZ GARCÍA, Miguel Ángel (2005): “Hacia un cártel perfecto. Los acuerdos colusivos en el sector siderúrgico español (1871-1907)", Investigaciones de Historia Económica, 1, pp. 131-161.

SÁNCHEZ RECIO, Glicerio, y TASCÓN FERNÁNDEZ, Julio (eds.) (2003): Los empresarios de Franco. Política y economía en España, 1936-1957, Barcelona, Crítica-Publicaciones Universidad de Alicante

TREFILERÍAS QUIJANO (1998): 125 Aniversario de Trefilerías Quijano, S.A. (18731998), Santander, Cámara Oficial de Comercio, Industria y Navegación de Cantabria.

VALDALISO, Jesús M. (1999): "Orígenes y desarrollo de la historia empresarial en España", Príncipe de Viana. Suplemento de Ciencias Sociales, 17, pp. 91-117.

WASSERMAN, Stanley, y FAUST, Catherine (1994): Social Network Analysis: Methods and Applications, New York, Cambridge University Press. 